\title{
Tumor hypoxia and reoxygenation: the yin and yang for radiotherapy
}

\author{
Beom-Ju Hong, BS*, Jeongwoo Kim, BS*, Hoibin Jeong, BS, Seoyeon Bok, BS, \\ Young-Eun Kim, BS, G-One Ahn, PhD \\ Division of Integrative Biosciences and Biotechnology, Pohang University of Science and Technology, Pohang, Korea
}

Tumor hypoxia, a common feature occurring in nearly all human solid tumors is a major contributing factor for failures of anticancer therapies. Because ionizing radiation depends heavily on the presence of molecular oxygen to produce cytotoxic effect, the negative impact of tumor hypoxia had long been recognized. In this review, we will highlight some of the past attempts to overcome tumor hypoxia including hypoxic radiosensitizers and hypoxia-selective cytotoxin. Although they were (still are) a very clever idea, they lacked clinical efficacy largely because of 'reoxygenation' phenomenon occurring in the conventional low dose hyperfractionation radiotherapy prevented proper activation of these compounds. Recent meta-analysis and imaging studies do however indicate that there may be a significant clinical benefit in lowering the locoregional failures by using these compounds. Latest technological advancement in radiotherapy has allowed to deliver high doses of radiation conformally to the tumor volume. Although this technology has brought superb clinical responses for many types of cancer, recent modeling studies have predicted that tumor hypoxia is even more serious because 'reoxygenation' is low thereby leaving a large portion of hypoxic tumor cells behind. Wouldn't it be then reasonable to combine hypoxic radiosensitizers and/or hypoxia-selective cytotoxin with the latest radiotherapy? We will provide some preclinical and clinical evidence to support this idea hoping to revamp an enthusiasm for hypoxic radiosensitizers or hypoxia-selective cytotoxins as an adjunct therapy for radiotherapy.

Keywords: Hypoxia, Radiosensitizers, Hypoxia-selective cytotoxin, Reoxygenation, Hyperfractionation, Hypofractionation

\section{Introduction}

Tumor hypoxia is a common feature in many, if not all, human and animal solid tumors, which can occur by two mechanisms: diffusion-limited chronic hypoxia, proposed by Thomlinson and Gray [1] more than 50 years ago where they observed uniform bands of necrotic cancer cells approximately 100-150 $\mu \mathrm{m}$ distant away from blood vessels in human tumors and perfusion-limited acute hypoxia proposed by Brown [2] in 1970's, which is caused by temporary obstruction or variable blood flow in tumor vessels (Fig. 1). By utilizing the goldstandard Eppendorf probe [3], the partial pressure for oxygen has been shown to be much lower in many types of solid tumors compared to that of the adjacent normal tissues [4]. It is important to note that there is no linear relationship between tumor size and the extent of hypoxia. A preclinical

Received 1 November 2016, Revised 11 December 2016, Accepted 12 December 2016.

Correspondence: G-One Ahn, PhD, Division of Integrative Biosciences and Biotechnology, Pohang University of Science and Technology, 77 Cheongam-ro, Nam-gu, Pohang 37673, Korea. Tel: +82-54-279-2353, Fax: +82-54-279-8379, E-mail: goneahn@postech.ac.kr

*Beom-Ju Hong and Jeongwoo Kim are contributed for this work equally.

(c) This is an Open Access article distributed under the terms of the Creative Commons Attribution Non-Commercial License (http://creativecommons.org/ licenses/by-nc/4.0/) which permits unrestricted non-commercial use, distribution, and reproduction in any medium, provided the original work is properly cited.

www.e-roj.org 


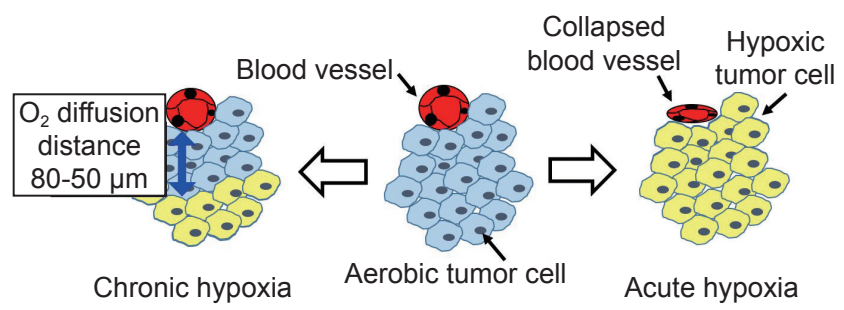

Fig. 1. A diagram demonstrating chronic hypoxia (left) and acute hypoxia (right).

study in human colorectal adenocarcinoma xenografts has reported that small tumors of less than $1 \mathrm{~mm}$ in diameter are already being extensively hypoxic and poorly perfused [5].

It has been extensively demonstrated that tumor hypoxia is a major contributing factor for the failure of many anticancer therapies such as surgery [6], chemotherapy [7], and radiotherapy [4]. Tumor hypoxia may also interfere with the efficacy of the latest anticancer immunotherapy. Many functions including differentiation, maturation, and proliferation of various populations of immune cells are known to be negatively affected by hypoxic conditions [8]. Furthermore, it is recently reported that the expression of programmed death-ligand 1 (PD-L1), the major inhibitory ligand towards cytotoxic T cells by binding to its cognate receptor PD-1 [9] is regulated by hypoxia-inducible factor-1 (HIF-1) [10], a master transcription factor well known to be stabilized in hypoxic cancer cells activating various pathways including angiogenesis, glycolytic metabolism, and metastasis [11].

Hypoxia is a direct and negative impact factor for radiotherapy because the molecular oxygen $\mathrm{O}_{2}$ is absolutely necessary to chemically fix DNA free radicals produced by

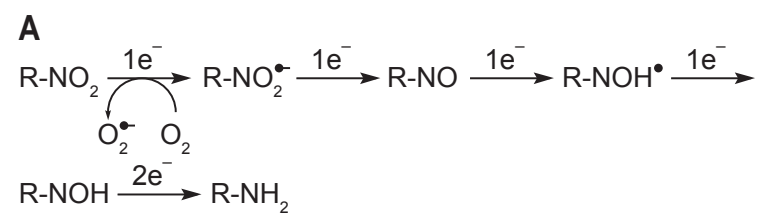

B

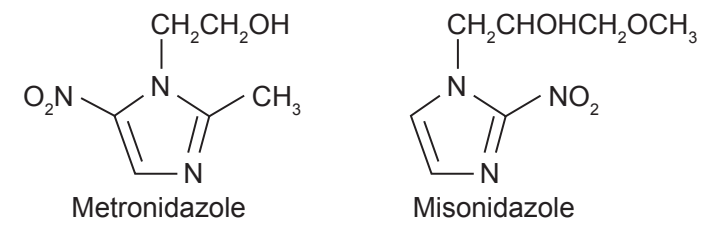

Fig. 2. Hypoxic radiosensitizers. (A) Hypoxic selective mechanism of action for nitroimidazole class $\left(\mathrm{R}-\mathrm{NO}_{2}\right)$ of radiosensitizers. (B) Chemical structure of metronidazole and misonidazole. ionizing radiation [4]. In the absence of $\mathrm{O}_{2}$, DNA radicals are repaired by abstracting hydrogen from sulfhydryl (SH) group present in protein [4]. It has been reported that three times higher ionizing radiation dose is required to kill hypoxic cancer cells compared to well-oxygenated cells in order to achieve the equivalent level of cell kill [4]. Because of this negative effect exerted by hypoxia on radiotherapy outcome, much attempts had previously been made to overcome tumor hypoxia. In this review, we will highlight some of such attempts and discuss future perspectives of tumor hypoxia for radiotherapy.

\section{Past Attempts with Hypoxic Radiosensitizers}

One of the earliest attempts to overcome radioresistance mediated by hypoxic tumor cells was to increase oxygen levels in the blood stream by making patients breathe 100\% oxygen at 3 atmosphere pressure [12]. However, clinical results were mixed and the reason was suggested because this strategy was ineffective in controlling acutely hypoxic cells [13]. In some cases, the use of carbogen $\left(95 \% \mathrm{O}_{2} / 5 \% \mathrm{CO}_{2}\right)$ has demonstrated a greater benefit than 100\% oxygen in increasing oxygen levels in the blood because of vasodilation effect exerted by $\mathrm{CO}_{2}$ [14]. Other approaches were the use of nicotinamide, an agent to increase tumor blood flow [15], artificial blood substitutes carrying increased levels of oxygen such as perfluorocarbons [16], and hyperthermia [17] although none of these treatments were proven to be clinically effective.

In 1960's Adams and Cooke [18 ] proposed a clever idea that electron-affinic chemical drugs may act like $\mathrm{O}_{2}$, a potent radiosensitizer. Radiosensitizer would accept thereby react with an electron (Fig. 2A) such as DNA radical produced by ionizing radiation. Radiosensitizers would exert their hypoxic selective mode of action because the chemical reduction would not occur in the presence of $\mathrm{O}_{2} . \mathrm{O}_{2}$ either strongly back-oxidizes this reductive chemical reaction (Fig. 2A) or reacts with DNA radical faster than radiosensitizers would. However, unlike $\mathrm{O}_{2}$, these agents are not being metabolized by cells through which they penetrate thus able to diffuse beyond the oxygen diffusion distance [19]. With misonidazole and metronidazole being the prototype members of nitroimidazole class of hypoxic radiosensitizers (other classes of radiosensitizers are reviewed elsewhere by Ahn and Brown [20]) (Fig. 2B), many clinical studies were conducted but revealed rather disappointing results. They had very little efficacy in improving the clinical response while there was a high incidence of peripheral neuropathy when multiple doses 
were given [21]. Why multiple doses? Repeated administration of radiosensitizers was required because it needed to be given immediately prior to each fraction of $2 \mathrm{~Gy}, 5$ fractions per week for 4-8 weeks of the conventional 'fractionated' schedule of radiotherapy. Therefore attempts to increase hydrophilicity thereby lowering peripheral neuropathy of misonidazole had been made and this lead to the development of analogs including etanidazole [22], pimonidazole [23], and nimorazole [19]. Although etanidazole was shown to be significantly less toxic in mice [22] as with misonidazole it caused peripheral neuropathy upon chronic administration [24] and it lacked a significant clinical efficacy in a randomized phase III trial in head and neck cancer patients treated with radiotherapy [25]. Pimonidazole demonstrated approximately 10 -fold increase in radiosensitization potency in vitro compared to misonidazole [26] although its dose limiting toxicity was still associated with some immediate effects involving the central nervous system [23]. As with other radiosensitizers, pimonidazole was not effective as an adjunct to radiotherapy and one of the reasons had been suggested to be due to an incomplete randomization of patients between control and test arms such that there were unexpectedly good results obtained in the control arm of the trial [27]. Despite all these disappointing clinical results above, a recent meta-analysis performed in 4,805 head and neck cancer patients in 32 randomized clinical trials revealed that hypoxic modifications such as oxygen breathing, the use of nicotinamide or nitroimidazoles (misonidazole, metronidazole, and etanidazole) offered a significant clinical benefit when loco-regional control and overall survival were used as the endpoint [28]. With the exhausted enthusiasm for hypoxic radiosensitizers, misonidazole is currently being utilized in the clinic as a positron-emission tomography (PET) [29] probe detecting tumor hypoxia [30] and pimonidazole, also known as 'hypoxyprobe' is being used to detect the tissue hypoxia in the pre-clinical setting [31]. Doranidazole is the latest member of this class of hypoxic radiosensitizers and has recently shown a significant improvement in the long-term survival of unresectable pancreatic cancer patients when given with 25 Gy postoperative radiotherapy [32].

In the mid 80's, Brown [33] developed a novel agent named tirapazamine that can selectively kill hypoxic cells thereby turning tumor hypoxia from a problem to a selective treatment advantage (Fig. 3). This so-called 'hypoxia-selective cytotoxin' tirapazamine demonstrated hypoxic cytotoxicity ratio of 50 200 in murine and human cancer cell lines although in vivo hypoxic cytotoxicity was somewhat lower than that in vitro [34]. Tirapazamine had been extensively tested in combination

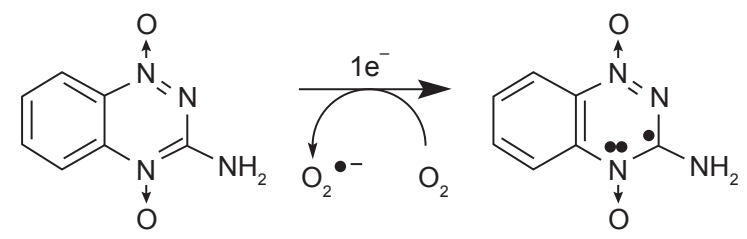

Fig. 3. Hypoxic selective mechanism of action for tirapazamine.

with radiation or cisplatin in many preclinical and clinical studies [34]. With the dose-limiting toxicities of reversible muscle cramping, nausea, and vomiting, many phase I and II trials demonstrated promising results in patients particularly with the head and neck or the lung cancers [34]. However, despite promising earlier clinical results, most of the phase III clinical trials results turned out that tirapazamine did not add a significant improvement in prolonging the overall survival for patients treated with chemotherapy [34] or chemoradiation [29], likely to be due to the lack of information on the extent of patients' tumor hypoxia upon patient selection and randomization of the trial.

\section{Hypoxia Imaging-Important Lessons We Have Recently Learned from ${ }^{18} \mathrm{~F}$-MISO and Tirapazamine}

Although misonidazole lacked clinical efficacy as a radiosensitizer, ${ }^{18} \mathrm{~F}$-misonidazole $\left({ }^{18} \mathrm{~F}\right.$-MISO) is currently being utilized in the clinic as a PET probe imaging tumor hypoxia [35]. With recent ${ }^{18} \mathrm{~F}$-MISO PET imaging studies, we have acquired some very valuable information.

First, Trans-Tasman Radiation Therapy Oncology Group (RTOG) 98.02 study in advanced squamous cell carcinoma of the head and neck patients has demonstrated that tirapazamine, which had otherwise failed in phase III clinical trials above [29] could be dramatically effective in lowering the locoregional failure if patients were selected based on tumor hypoxia by ${ }^{18} \mathrm{~F}$-MISO PET scans in prior [36]. In that study, only one of 19 head and neck cancer patients had recurrence when tirapazamine was given in combination with chemoradiation whereas 8 out of 13 patients recurred if they received 5-fluorouracil in place of tirapazamine [36] (Fig. 4). These results thus indicate that all those clever ideas developed in 1970's and 1980's would work well if we could better identify patients with significant hypoxic fractions being present in their tumors.

Second, ${ }^{18}$ F-MISO PET imaging has shown that tumor hypoxia can dramatically change over time, as early as a few 


\begin{tabular}{|c|c|c|c|c|}
\hline \multicolumn{5}{|c|}{ Locoregional Failure by Treatment Arm and Hypoxia } \\
\hline & \multicolumn{4}{|c|}{ Treatment } \\
\hline & \multicolumn{2}{|c|}{ Chemoboost } & \multicolumn{2}{|c|}{ TPZ / CIS } \\
\hline Hypoxia Status & $\begin{array}{c}\text { No. of } \\
\text { Locoregional } \\
\text { Failures }\end{array}$ & $\begin{array}{c}\text { No. of } \\
\text { Patients }\end{array}$ & $\begin{array}{c}\text { No. of } \\
\text { Locoregional } \\
\text { Failures }\end{array}$ & $\begin{array}{c}\text { No. of } \\
\text { Patients }\end{array}$ \\
\hline Nonhypoxic & 1 & 10 & 2 & 3 \\
\hline $\begin{array}{l}\text { Hypoxia in primary tumor } \\
\text { and / or nodes }\end{array}$ & 8 & 13 & 1 & 19 \\
\hline
\end{tabular}

- CIS $\left(50 \mathrm{mg} / \mathrm{m}^{2}\right)+\mathrm{RT}$ (week 6\&7)

- Cont. 5-FU $\left(360 \mathrm{mg} / \mathrm{m}^{2} / \mathrm{d}\right)$ d1-5 in week 6\&7)

RT: 70 Gy in 35 fractions over 7 weeks
- TPZ $\left(290 \mathrm{mg} / \mathrm{m}^{2}\right)+\mathrm{CIS}(50$ $\left.\mathrm{mg} / \mathrm{m}^{2}\right)+\mathrm{RT}$ (week 6\&7)

- TPZ $\left(160 \mathrm{mg} / \mathrm{m}^{2}\right)$ week $2 \& 3$

Fig. 4. Locoregional failure by treatment arm and hypoxia. Tirapazamine offers a significant benefit lowering the locoregional failures in head and neck cancer patients with hypoxia (red box). TPZ/CIS, tirapazamine/cisplatin; RT, radiotherapy. Modified from Rischin et al. [36] with permission of the American Society of Clinical Oncology.

A
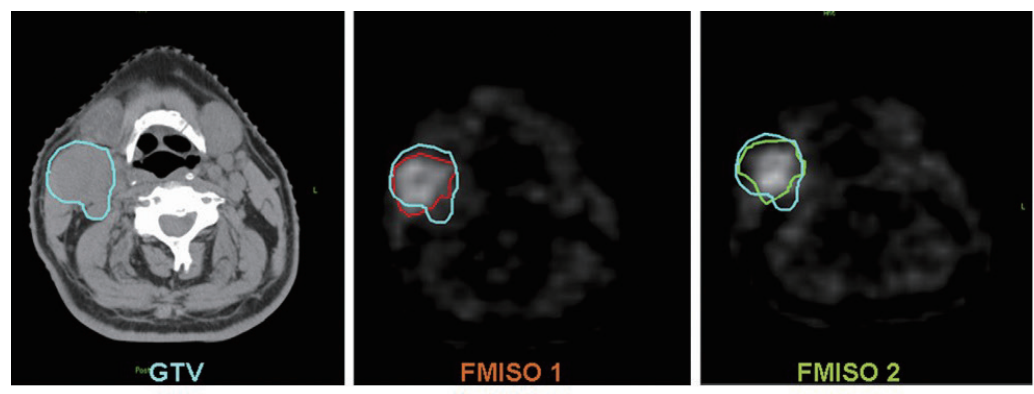

B
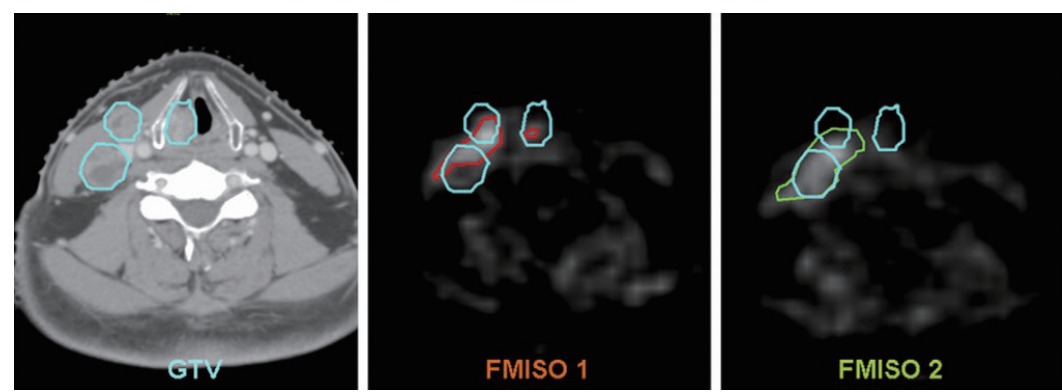
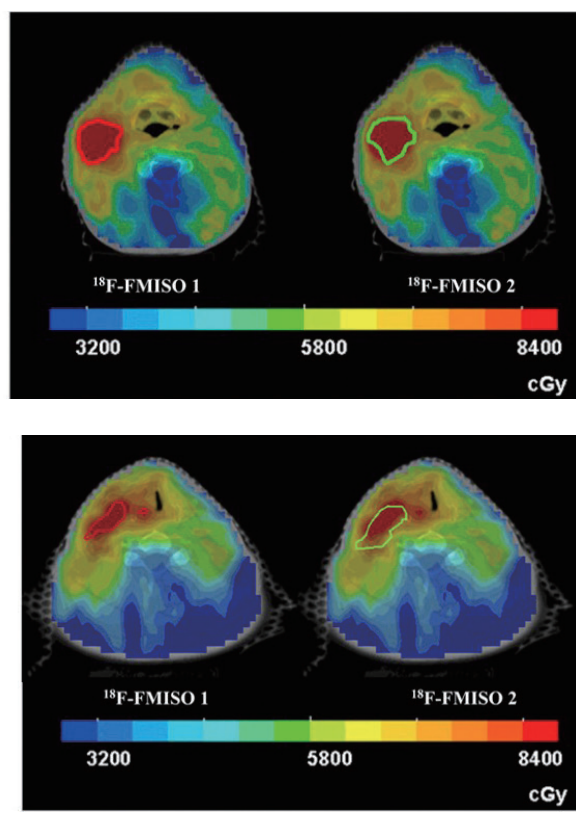

Fig. 5. Tumor hypoxia is dynamically changing. (A) A positron emission tomography-computed tomography (PET-CT) image (left) and intensity-modulated radiotherapy (IMRT) treatment (right) for a patient whom do not demonstrate a significant change in the tumor hypoxia as examined by two consecutive ${ }^{18} \mathrm{~F}$-misonidazole $\left({ }^{18} \mathrm{~F}-\mathrm{MISO}\right)$ images (left). (B) A significant change in the location of tumor hypoxia is noted in this patient between 1st (red area) and 2nd (green area) images of F-MISO PET (left). Upon IMRT treatment (right), newly hypoxic areas (green) would receive the dose not enough to kill hypoxic cells while those tumor areas that are no longer hypoxic would experience overdose of irradiation. Adapted from Lin et al. [38] with permission of Elsevier.

hours up to days [37]. Although there had been some attempts to measure the dynamics and kinetics of changes in tumor hypoxia, it was largely concluded that it occurred rather subtly and unpredictably, and that it frequently associated with immature blood vessels [37]. Due to the unpredictable nature, changes in tumor hypoxia can significantly complicate the latest radiotherapy technology, for example intensitymodulated radiotherapy (IMRT; see below 'Recent advances in radiotherapy technology') dose-painting technology where additional boost dose of irradiation can be given to hypoxic 
regions of tumors. For example, Lin et al. [38] have reported that some patients whose tumor hypoxia varies significantly from day to day would not benefit such dose-painting radiotherapy because 'newly hypoxic' regions of tumors would receive the dose that is not high enough to kill hypoxia while 'now no-longer' hypoxic regions would be treated with unnecessarily high dose of irradiation (Fig. 5).

\section{Recent Advances in Radiotherapy Technologies}

The ways to deliver radiotherapy have been drastically changed over the last decades. Previously, conventional radiotherapy had been consisted of relatively small doses (1.2-3 Gy/ fraction) daily fraction over weeks or months based on a small survival advantage of the normal tissues over the tumors [39]. Importantly, this treatment regimen has led to 'reoxygenation' phenomenon, in which surviving hypoxic tumor cells at a given dose become oxygenated by the time of the next fractionation treatment [40], which has turned out to be one way to effectively kill hypoxic tumor cells. Much of radiobiological work with this fractionated irradiation has taught us that the normal tissue toxicity occurring by fractionated irradiation has been shown to be dependent upon several factors including the fraction size, tissue type, and total doses of radiation [39]. Based on this understanding, some of altered fractionation schemes were further developed: hyperfractionation of smaller fraction doses, increased numbers of fractions, and interfractionation intervals by at least 6 hours (because sublethal damage repair has shown to occur within 6 hours); or accelerated fractionation where the overall treatment time being shortened in order to reduce accelerated repopulation of tumor cells [39]. These modifications in fractionated irradiation have shown to improve the local control rates while the late normal tissue toxicity not being significantly different [41].

Recent technological advancement has allowed us to deliver single large fraction of 15-20 Gy precisely to the tumorbearing volume, known as stereotactic radiosurgery (SRS) for cranial lesions and stereotactic body radiotherapy (SBRT) for other extracranial sites [39]. Use of such technologies has significantly improved the clinical response: for example recent results from RTOG 0236 protocol in non-operable earlystage lung cancer patients treated with 3 fractions of $20 \mathrm{Gyl}$ fraction SBRT have shown 3 year local control and survival to be $98 \%$ and $56 \%$, respectively, the results being comparable to surgery [42]. Computer-controlled beam shapes with multileaf collimators and intensity modification by using a wedge- shaped compensating filter have led to IMRT allowing highly conformal distributions of high doses of radiation in target volumes while maintaining the low levels of radiation to the surrounding normal tissues [39].

\section{Tumor Hypoxia with SBRT-Back to Square One?}

Without a doubt, the recent advancement in radiotherapy technology has brought superior clinical responses in numerous cancer patients. However has this technology overcome the problem of tumor hypoxia? A recent modeling study by Carlson et al. [43] suggested that assuming daily fractionation and full reoxygenation between fractions, tumor hypoxia is actually more serious problem with SBRT. Tumor cell survival can increased up to 100-fold as we choose 'less number' of fractions and 'at higher dose' per fraction (' $A$ ' in Fig. 6), which may be equivalent to current SBRT. In addition, an increase in hypoxic fraction from 10\% to 30\% has modeled to reflect nearly 10 times further resistance of tumor cells at

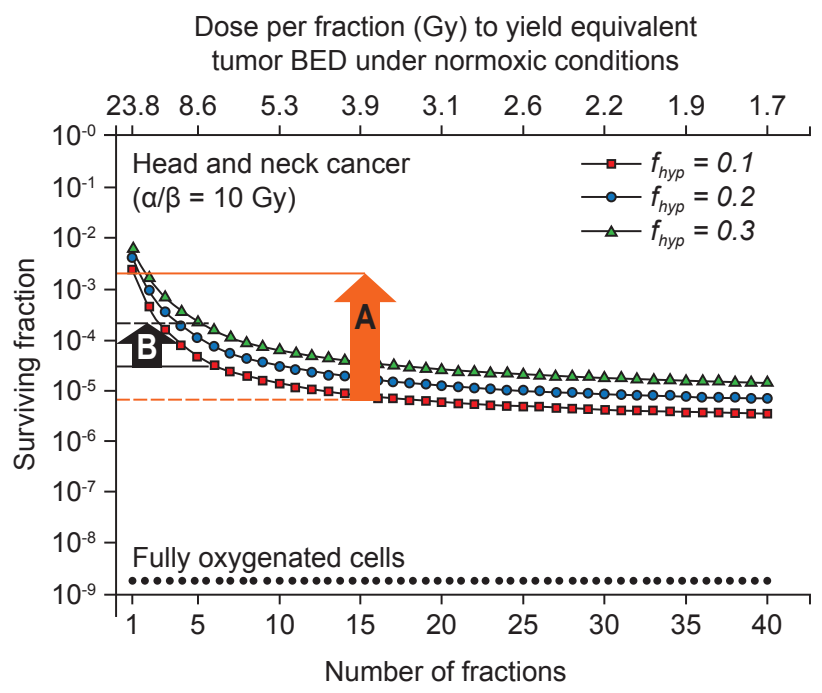

Fig. 6. Total surviving clones as a function of dose per fraction assuming daily fractionation and full reoxygenation between fractions. A: For tumors with $10 \%$ hypoxia $\left(f_{\text {hyp }}=0.1\right.$; red line) there is more than 2 logs (100-fold) of cell survival advantage (pointed with the orange arrow) as we reduce the number of fractionations (as we shift right to left). B: As hypoxic fraction increases from $10 \%\left(f_{\text {hyp }}=0.1\right.$; red line $)$ to $30 \%\left(f_{\text {hyp }}=0.3\right.$; green line), there is additional $1 \log (10$-fold) of cell survival increase (pointed with the black arrow) at 5 fractionation treatment, at which the cell survival is already being much higher than that by hyperfractionation. Adapted from Carlson et al. [43] with permission of Elsevier. 
this high dose hypofractionated regimen [43] ('B' in Fig. 6). The reason has been suggested largely to be due to a reduction in interfractionation reoxygenation associated with this single high dose SBRT.

What can we do about it? An obvious way would be to deliver the next fraction of SBRT to tumors where the tumor reoxygenation would be at its maximum. This may even mean 'personalized' radiotherapy based on monitoring individuals' changes in tumor hypoxia, which in reality would be extremely difficult to do so. In fact, one of current dilemma for SBRT is that there are no standardized intervals between each hypofractionation. For examples, Timmerman et al. [44] prescribed 18 Gy per fraction $\times 3$ fractions within 2 weeks, while Nagata et al. [45] prescribed 12 Gy per fraction $\times 4$ over 5-13 days. In one study 50 Gy was delivered over 4 consecutive days, meaning 12.5 Gy every day for 4 days [46]. Surprisingly all those studies demonstrated good clinical outcome despite differences in their fractionation intervals. Does it mean that SBRT does not rely on reoxygenation? Probably the good clinical response may derive from such 'ablative' doses of ionizing radiation producing tremendous of DNA damages in the affected areas. We have recently investigated if we can determine changes in tumor hypoxia before and after a single high dose of 20 Gy irradiation by utilizing various techniques including ${ }^{18} \mathrm{~F}$-MISO PET imaging, pimonidazole, and bioluminescence imaging in a mouse tumor model [47]. We found that tumor hypoxia did not significantly change when examined at 6 hours, 2 days, and 5 days postradiation although we interestingly observed that such high dose irradiation caused a rapid but transient vascular collapse [47]. There are similar attempts currently undergoing in lung cancer patients by Kelada et al. [48]. In their preliminary study, there was an increase in tumor hypoxia shortly after 18 Gy measured by ${ }^{18} \mathrm{~F}$-MISO PET imaging although inter- and intra-patient variability was too high to firmly conclude their findings.

If inter-and intra-individual tumor hypoxia changes are too variable to predict, an easy alternative (at least until we have a better technology imaging/monitoring real time changes of tumor hypoxia) would be to use radiosensitizers. As a matter of fact, radiosensitizers of many preclinical studies of in vivo $[49,50]$ and in vitro [51] in earlier days had been tested at much higher radiation doses (5-30 Gy), equivalent to today's SBRT and demonstrated that it would work much better with high doses of irradiation [51] (Fig. 7). A recent preclinical study by Wittenborn and Horsman [52] has demonstrated the proofof-concept that $\mathrm{TCD}_{50}$ (radiation dose to cure $50 \%$ of tumors) can be decreased from 29.7 Gy to 2.5 Gy when $3 \times 15$ Gy was combined with nimorazole 30 minutes prior to each fraction (Table 1). Clinical enthusiasm may have been exhausted and doomed because of disappointing clinical results with nearly all of hypoxic radiosensitizers tested (see above 'Past attempts with hypoxic radiosensitizers'). However, there is a clinical study as early as in 1980's that 9 out of 10 vulvovaginal cancer patients achieved the local control when metronidazole was given as a radiosensitizer with high-dose radiation [53]. A recent clinical study with doranidazole has demonstrated a significant improvement in 3-year survival in advanced
A

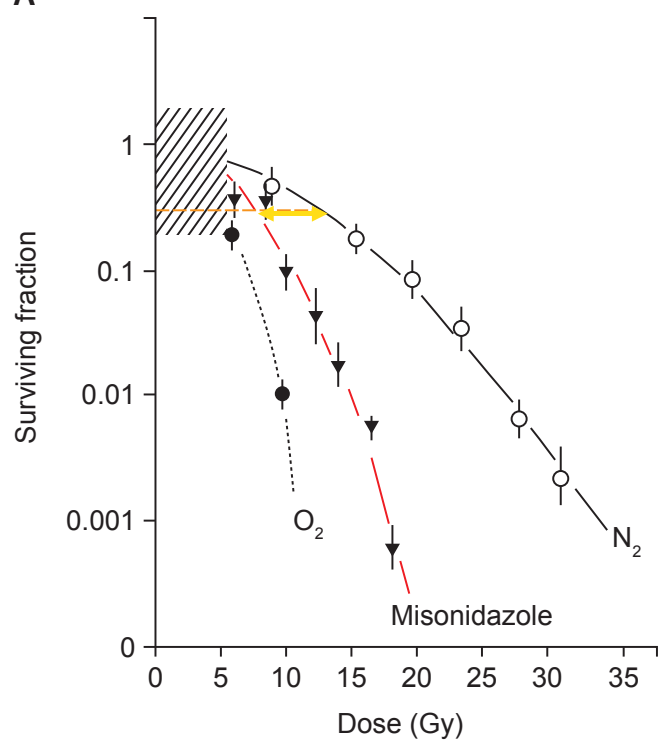

B

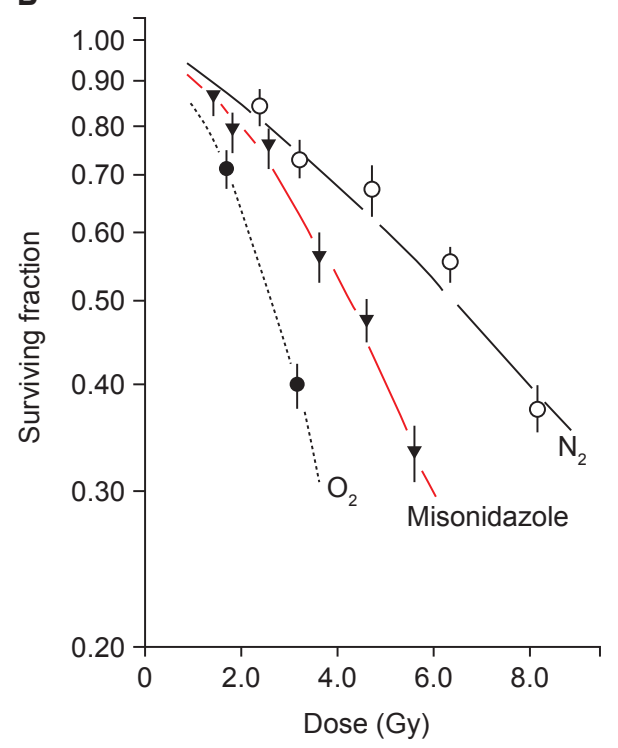

Fig. 7. Survival curves of Chinese hamster ovary $(\mathrm{CHO})$ cells irradiated under oxic $\left(\mathrm{O}_{2}\right)$, hypoxic $\left(\mathrm{N}_{2}\right)$, or hypoxic conditions in the presence of $1.5 \mathrm{mM}$ misonidazole (red line) by 270 $\mathrm{kVp}$ X-ray irradiation at (A) high or (B) low dose range. Note that sensitization enhancement ratio (yellow arrows) of misonidazole is larger at higher irradiation dose range (A). Adapted from Palcic et al. [51] with permission of publisher. 
Table 1. Tumor control dose $50\left(\mathrm{TCD}_{50}\right)$ for radiation only or radiation combined with hypoxic targeting agent(s) in mice

\begin{tabular}{lc}
\hline \multicolumn{1}{c}{ Treatment $^{\mathrm{a})}$} & $\mathrm{TCD}_{50}{ }^{\mathrm{b})}( \pm 95 \% \mathrm{Cl})$ \\
\hline Radiation alone $(3 \times 15 \mathrm{~Gy})$ & $29.7(22.9-38.5)$ \\
Radiation $+1 \times$ nimorazole & $20.1(11.7-34.3)$ \\
Radiation $+3 \times$ nimorazole & $2.5(0.5-13.7)^{*}$ \\
Radiation $+1 \times$ nimorazole and & $22.9(16.2-32.4)$ \\
$\quad$ carbogen & \\
Radiation $+3 \times$ nimorazole and & $<1.0$ (not measurable) \\
carbogen & $12.2(6.0-24.9)^{*}$ \\
Radiation $+1 \times$ OXi4503 & $12.1(7.9-18.6)^{*}$ \\
Radiation $+3 \times$ OXi4503 & $10.1(7.0-14.6)^{*}$ \\
Radiation $+1 \times$ hyperthermia & $8.6(4.5-16.6)^{*}$ \\
Radiation $+3 \times$ hyperthermia & $1.9(0.4-8.5)^{*}$ \\
Radiation $+1 \times$ OXi4503 and & \\
hyperthermia & \\
\hline
\end{tabular}

The study showing that tumor control dose $50\left(\mathrm{TCD}_{50}\right)$ can dramatically be reduced when high dose (15 Gy) irradiation is combined with hypoxic modifications such as nimorazole (Radiation alone), nicotinamide (Radiation $+3 \times$ nimorazole) and carbogen (Radiation $+3 \times$ nimorazole and carbogen), and hyperthermia. $\mathrm{Cl}$, confidence interval.

${ }^{a}$ All modifiers were given with either each $15 \mathrm{~Gy}$ fraction $(3 \times)$ or only the last $15 \mathrm{~Gy}$ fraction $(1 \mathrm{x}) .{ }^{\mathrm{b})} \mathrm{TCD} \mathrm{D}_{50}$, clamped top-up radiation dose needed to control $50 \%$ of the tumors. Data were obtained from Figures 1 and 2 [52].

*indicates a statistically significant difference when compared to radiation alone $(p<0.05)$.

Adapted from Wittenborn and Horsman [52] with permission of Taylor \& Francis.

pancreatic cancer patients when it was combined with $25 \mathrm{~Gy}$ intraoperative radiotherapy [32]. These preclinical and clinical results thus indicate that hypoxic radiosensitizers or hypoxiaselective cytotoxins may work well with the latest high dose hypofractionation radiotherapy.

\section{Future Perspectives}

Because of tumor hypoxia and the normal tissue complication, other radiotherapy modalities such as charged particle beam radiotherapy of proton or carbon ion are being actively developed and utilized to treat cancers world-wide. Compared to X-ray, carbon ion offers advantages of superior cell kill and a potential to decrease normal tissue toxicity [54]. This is first because carbon ion is of high linear energy transfer (LET), it does not depend on the oxygen effect for producing cell kill. Second, Bragg peak, the extent of energy loss of radiotherapy along the penetration depth of tissues as it travels through is even optimal with carbon ion therapy among other heavier ions-carbon ion has the lowest entry relative biological effectiveness (RBE; the ratio in the dose of one type of ionizing radiation to another at the same level of biological effects) while it demonstrates the maximal RBE in Bragg peak [54]. Some of the current limitation of this approach may include a limited availability of the facility, high treatment costs, and rather short clinical history compared to X-ray ionizing radiation.

In an attempt to take an advantage of the existing clinical linear accelerator (LINAC), the newest effort has been suggested to use ultra-high dose rate FLASH irradiation [55]. The concept was first introduced by Favaudon et al. [56] demonstrating that the ultra-high dose rates in the range of 40-60 Gy per second resulted in a significantly reduced lung toxicity while the local control of tumors was similar compared to conventional 17 Gy irradiation. Recently, Schuler et al. [55] are actively tuning and modifying a clinical LINAC instrument for electron FLASH offering an easy set up and more homogenous dose distribution in tumors than photoninduced radiotherapy.

Optical approaches in imaging oxygen tensions and delivering oxygen to hypoxic regions of tumors are also being actively developed in the preclinical settings. Studies have shown that redox ratio of autofluorescence for endogenous molecules such as hemoglobin could reflect the oxygen tensions in tumors [57] and that this can predict the tumor response to irradiation in FaDu head and neck squamous cell carcinoma xenograft models in mice [58]. This approach may be limited to determination of the oxygen partial pressure in blood vessels of tumors not necessarily reflecting those within tumors. In this respect, we have recently developed an optical oxygen sensor consisting of ruthenium ( $R u$ ) complex encapsulated in amphiphilic polymer where we can quantify the oxygen concentrations based on the ratiometric analyses of Ru phosphorescence signal in cancer cells [59]. Dewhirst group has also reported a similar approach of boron nanoparticle where they have quantified the oxygen in tumors implanted underneath in vivo dorsal window chamber [60]. A platinum complex of porphyrin complexes has recently demonstrated low yet heterogeneous oxygen tension distributions in the bone marrow of mice by using phosphorescence lifetime imaging [61]. Nanoparticle approaches delivering the molecular oxygen to tumors are also very exciting. Prasad et al. [62] have shown that albumin-coated manganese oxide nanoparticles release the molecular oxygen by $\mathrm{H}_{2} \mathrm{O}_{2}$ produced by oxidative stress within the tumor microenvironment, which can increase the tumor oxygenation up to $45 \%$ in tumors leading 


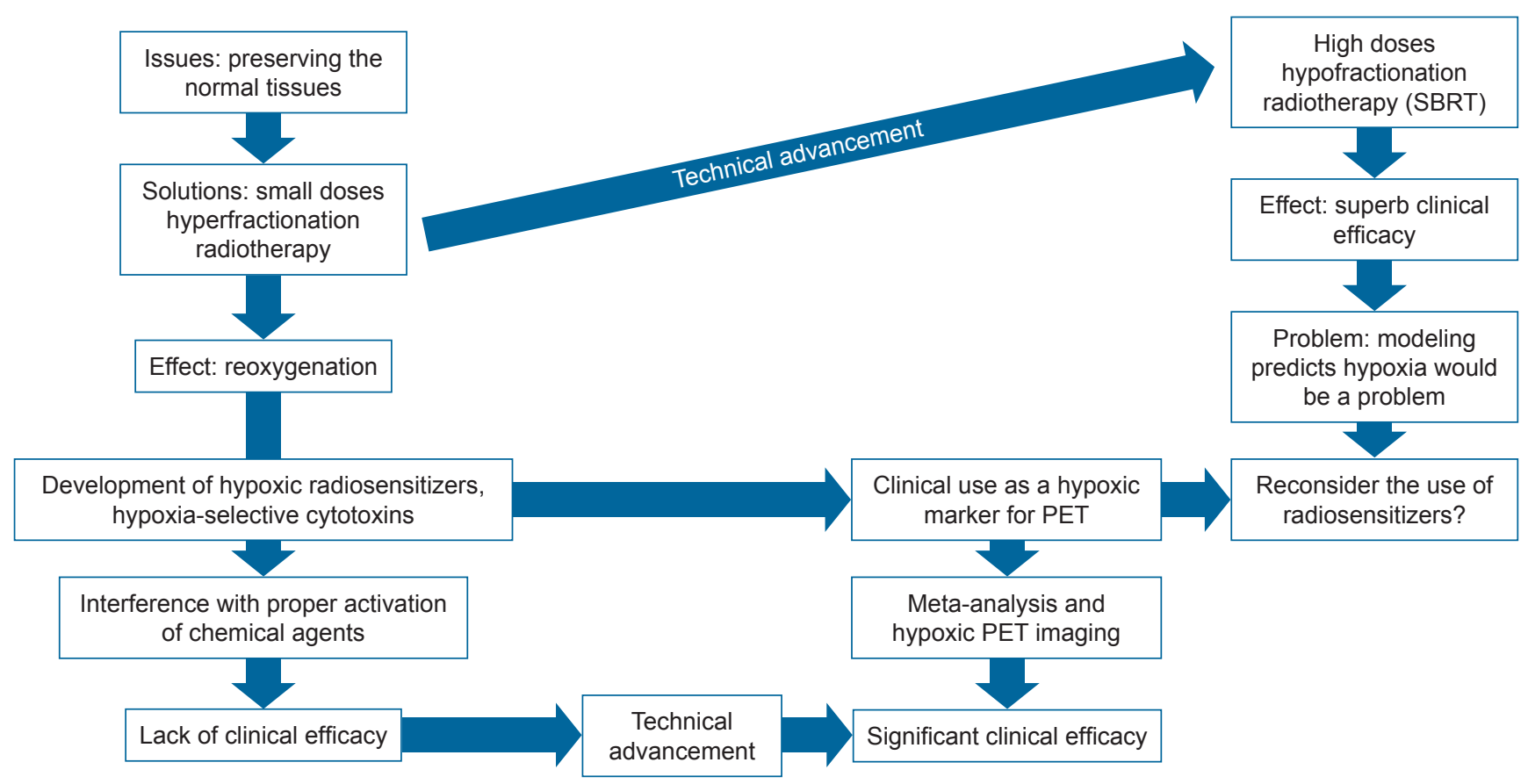

Fig. 8. A diagram demonstrating development of hypoxic radiosensitizers and hypoxia-selective cytotoxins. Reoxygenation by the conventional low doses hyperfractionation radiotherapy had previously been interfered with proper activation of those compounds in tumors. Because interfractionation reoxygenation is now being predicted to be low with the latest high doses hypofractionation radiotherapy stereotactic body radiotherapy (SBRT), perhaps it is sufficiently reasonable to combine hypoxic radiosensitizers or hypoxiaselective cytotoxins with such radiotherapy techniques. PET, positron-emission tomography.

to a significantly increased radiotherapy efficacy in breast cancer models in mice. Another interesting approach using nanoperfluorocarbon of high oxygen dissolving properties, Song et al. [63] have recently demonstrated that these agents can pick up the oxygen from the lung and selectively release it in tumors only by ultrasound stimulation in hyperoxic breathing mice, thereby improving the tumor response to radiotherapy and photodynamic therapy [63]. Future studies including investigation of pharmacokinetics of these agents would be warranted in order to move forward to be applied in the clinical setting.

\section{Conclusions}

Small doses of hyperfractionation regimen had implemented to lower the normal tissue toxicity produced by ionizing radiation, which has brought us an important radiobiological concept 'reoxygenation.' While it turned out to kill hypoxic tumor cells efficiently, it paradoxically acted as a major disadvantage towards our previous efforts for hypoxic radiosensitizers and hypoxia-selective cytotoxins, the two great ideas which may have worked so well otherwise. The latest technology advancement in radiotherapy using high doses of hypofractionation regimen has totally changed the picture of cancer treatment and brought superb clinical responses. However, recent modeling studies suggest that tumor hypoxia would be a significant problem with the latest radiotherapy because the interfractionation reoxygenation would be minimal. While we do not have reoxygenation phenomenon to interfere with hypoxic radiosensitizers and/or hypoxia-selective cytotoxins, this may be a perfect time to reconsider combining these with the latest radiotherapy technique (Fig. 8).

\section{Conflict of Interest}

No potential conflict of interest relevant to this article was reported.

\section{Acknowledgments}

This study was supported by Ministry of Science, ICT, and Future Planning, Korea (NRF-2015K2A1A2070880 and NRF2015M2B2A9029247); and Ministry of Education, Korea (BK21 Plus 10Z20130012243). Hong BJ (NRF-2013H1A2A1032808), 
Kim JW (NRF-2016H1A2A1908006), and Kim YE (NRF2012H1A2A1002871) are Global PhD fellowship recipients supported by Ministry of Science, ICT, and Future Planning, Korea. Jeong $\mathrm{H}$ is a Cheong-Am Science Fellow supported by POSCO Cheong-Am Foundation, Korea.

\section{References}

1. Thomlinson RH, Gray LH. The histological structure of some human lung cancers and the possible implications for radiotherapy. Br J Cancer 1955;9:539-49.

2. Brown JM. Evidence for acutely hypoxic cells in mouse tumours, and a possible mechanism of reoxygenation. $\mathrm{Br} J$ Radiol 1979;52:650-6.

3. Nozue $M$, Lee I, Yuan $F$, et al. Interlaboratory variation in oxygen tension measurement by Eppendorf "Histograph" and comparison with hypoxic marker. J Surg Oncol 1997;66:30-8.

4. Brown JM, Wilson WR. Exploiting tumour hypoxia in cancer treatment. Nat Rev Cancer 2004;4:437-47.

5. Li XF, Carlin S, Urano M, Russell J, Ling CC, O'Donoghue JA. Visualization of hypoxia in microscopic tumors by immunofluorescent microscopy. Cancer Res 2007;67:7646-53.

6. Brizel DM, Scully SP, Harrelson JM, et al. Tumor oxygenation predicts for the likelihood of distant metastases in human soft tissue sarcoma. Cancer Res 1996;56:941-3.

7. Hammond EM, Giaccia AJ. Hypoxia-inducible factor-1 and p53: friends, acquaintances, or strangers? Clin Cancer Res 2006;12:5007-9.

8. Jeong H, Bok S, Hong BJ, Choi HS, Ahn GO. Radiation-induced immune responses: mechanisms and therapeutic perspectives. Blood Res 2016;51:157-63.

9. Keir ME, Butte MJ, Freeman GJ, Sharpe AH. PD-1 and its ligands in tolerance and immunity. Annu Rev Immunol 2008;26:677-704.

10. Noman MZ, Desantis G, Janji B, et al. PD-L1 is a novel direct target of HIF-1alpha, and its blockade under hypoxia enhanced MDSC-mediated T cell activation. J Exp Med 2014;211:781-90.

11. Semenza GL, Wang GL. A nuclear factor induced by hypoxia via de novo protein synthesis binds to the human erythropoietin gene enhancer at a site required for transcriptional activation. Mol Cell Biol 1992;12:5447-54.

12. Dische S. Local tumour control and its effect upon survival of the patient. Australas Radiol 1983;27:181-5.

13. Chaplin DJ, Durand RE, Stratford IJ, Jenkins TC. The radiosensitizing and toxic effects of RSU-1069 on hypoxic cells in a murine tumor. Int J Radiat Oncol Biol Phys 1986;12:1091-5.

14. Rockwell S. Oxygen delivery: implications for the biology and therapy of solid tumors. Oncol Res 1997;9:383-90.

15. Hoskin PJ, Rojas AM, Bentzen SM, Saunders MI. Radiotherapy with concurrent carbogen and nicotinamide in bladder carcinoma. J Clin Oncol 2010;28:4912-8.

16. Riess JG. Understanding the fundamentals of perfluorocarbons and perfluorocarbon emulsions relevant to in vivo oxygen delivery. Artif Cells Blood Substit Immobil Biotechnol 2005;33:47-63.

17. Sun $X$, Xing L, Ling CC, Li GC. The effect of mild temperature hyperthermia on tumour hypoxia and blood perfusion: relevance for radiotherapy, vascular targeting and imaging. Int J Hyperthermia 2010;26:224-31.

18. Adams GE, Cooke MS. Electron-affinic sensitization. I: A structural basis for chemical radiosensitizers in bacteria. Int J Radiat Biol Relat Stud Phys Chem Med 1969;15:457-71.

19. Dische $S$. Chemical sensitizers for hypoxic cells: a decade of experience in clinical radiotherapy. Radiother Oncol 1985;3:97-115.

20. Ahn GO, Brown M. Targeting tumors with hypoxia-activated cytotoxins. Front Biosci 2007;12:3483-501.

21. Dische $S$, Saunders MI, Lee ME, Adams GE, Flockhart IR. Clinical testing of the radiosensitizer Ro 07-0582: experience with multiple doses. Br J Cancer 1977;35:567-79.

22. Brown JM, Yu NY, Brown DM, Lee WW. SR-2508: a 2-nitroimidazole amide which should be superior to misonidazole as a radiosensitizer for clinical use. Int J Radiat Oncol Biol Phys 1981;7:695-703.

23. Saunders MI, Anderson PJ, Bennett MH, et al. The clinical testing of Ro 03-8799: pharmacokinetics, toxicology, tissue and tumor concentrations. Int J Radiat Oncol Biol Phys 1984;10:1759-63.

24. Coleman CN, Hirst VK, Brown DM, Halsey J. The effect of vitamin B6 on the neurotoxicity and pharmacology of desmethylmisonidazole and misonidazole: clinical and laboratory studies. Int J Radiat Oncol Biol Phys 1984;10:1381-6.

25. Lee DJ, Cosmatos D, Marcial VA, et al. Results of an RTOG phase III trial (RTOG 85-27) comparing radiotherapy plus etanidazole with radiotherapy alone for locally advanced head and neck carcinomas. Int J Radiat Oncol Biol Phys 1995;32:567-76.

26. Smithen CE, Clarke ED, Dale JA, et al. Novel (nitro-1-imidazolyl) alkanolamines as potential radiosensitisers with improved therapeutic properties. In: Brady LW, editor. Radiation sensitizers: their use in the clinical management of cancer. New York: Masson Publishing Inc.; 1980. p. 22-32.

27. Dische $S$, Machin D, Chassange D. A trial of Ro 03-8799 (pimonidazole) in carcinoma of the uterine cervix: an interim report from the Medical Research Council Working Party on advanced carcinoma of the cervix. Radiother Oncol 1993;26:93-103.

28. Overgaard J. Hypoxic modification of radiotherapy in squamous cell carcinoma of the head and neck: a systematic 
review and meta-analysis. Radiother Oncol 2011;100:22-32.

29. Rischin D, Peters $\sqcup$, O'Sullivan $B$, et al. Tirapazamine, cisplatin, and radiation versus cisplatin and radiation for advanced squamous cell carcinoma of the head and neck (TROG 02.02, HeadSTART): a phase III trial of the Trans-Tasman Radiation Oncology Group. J Clin Oncol 2010;28:2989-95.

30. Wilson WR, Hay MP. Targeting hypoxia in cancer therapy. Nat Rev Cancer 2011;11:393-410.

31. Arteel GE, Thurman RG, Raleigh JA. Reductive metabolism of the hypoxia marker pimonidazole is regulated by oxygen tension independent of the pyridine nucleotide redox state. Eur J Biochem 1998;253:743-50.

32. Karasawa K, Sunamura M, Okamoto $A$, et al. Efficacy of novel hypoxic cell sensitiser doranidazole in the treatment of locally advanced pancreatic cancer: long-term results of a placebocontrolled randomised study. Radiother Oncol 2008;87:32630.

33. Brown JM. The hypoxic cell: a target for selective cancer therapy: eighteenth Bruce F. Cain Memorial Award lecture. Cancer Res 1999;59:5863-70.

34. Marcu L, Olver I. Tirapazamine: from bench to clinical trials. Curr Clin Pharmacol 2006;1:71-9.

35. Fleming IN, Manavaki R, Blower PJ, et al. Imaging tumour hypoxia with positron emission tomography. $\mathrm{Br} J$ Cancer 2015;112:238-50.

36. Rischin D, Hicks RJ, Fisher R, et al. Prognostic significance of [18F]-misonidazole positron emission tomography-detected tumor hypoxia in patients with advanced head and neck cancer randomly assigned to chemoradiation with or without tirapazamine: a substudy of Trans-Tasman Radiation Oncology Group Study 98.02. J Clin Oncol 2006;24:2098-104.

37. Dewhirst MW. Relationships between cycling hypoxia, HIF-1, angiogenesis and oxidative stress. Radiat Res 2009;172:65365.

38. Lin Z, Mechalakos J, Nehmeh S, et al. The influence of changes in tumor hypoxia on dose-painting treatment plans based on 18F-FMISO positron emission tomography. Int J Radiat Oncol Biol Phys 2008;70:1219-28.

39. Connell PP, Hellman S. Advances in radiotherapy and implications for the next century: a historical perspective. Cancer Res 2009;69:383-92.

40. Kallman RF. The phenomenon of reoxygenation and its implications for fractionated radiotherapy. Radiology 1972;105:135-42.

41. Fu KK, Pajak TF, Trotti A, et al. A Radiation Therapy Oncology Group (RTOG) phase III randomized study to compare hyperfractionation and two variants of accelerated fractionation to standard fractionation radiotherapy for head and neck squamous cell carcinomas: first report of RTOG 9003. Int J Radiat Oncol Biol Phys 2000;48:7-16.

42. Timmerman RD. Surgery versus stereotactic body radiation therapy for early-stage lung cancer: who's down for the count? J Clin Oncol 2010;28:907-9.

43. Carlson DJ, Keall PJ, Loo BW Jr, Chen ZJ, Brown JM. Hypofractionation results in reduced tumor cell kill compared to conventional fractionation for tumors with regions of hypoxia. Int J Radiat Oncol Biol Phys 2011;79:1188-95.

44. Timmerman R, Paulus R, Galvin J, et al. Stereotactic body radiation therapy for inoperable early stage lung cancer. JAMA 2010;303:1070-6.

45. Nagata Y, Takayama K, Matsuo Y, et al. Clinical outcomes of a phase I/II study of 48 Gy of stereotactic body radiotherapy in 4 fractions for primary lung cancer using a stereotactic body frame. Int J Radiat Oncol Biol Phys 2005;63:1427-31.

46. Chang JY, Balter PA, Dong L, et al. Stereotactic body radiation therapy in centrally and superiorly located stage I or isolated recurrent non-small-cell lung cancer. Int J Radiat Oncol Biol Phys 2008;72:967-71

47. Song $C$, Hong BJ, Bok $S$, et al. Real-time tumor oxygenation changes after single high-dose radiation therapy in orthotopic and subcutaneous lung cancer in mice: clinical implication for stereotactic ablative radiation therapy schedule optimization. Int J Radiat Oncol Biol Phys 2016;95:1022-31.

48. Kelada OJ, Decker RH, Zheng MO, et al. Quantification of tumor hypoxia in lung cancer patients undergoing stereotactic body radiotherapy using dynamic PET imaging. In: 15th International Congress of Radiation Research (ICRR) Annual Meeting; 2015 May 25-29; Kyoto, Japan.

49. Siemann DW, Hill SA. Increased therapeutic benefit through the addition of misonidazole to a nitrosourea-radiation combination. Cancer Res 1986;46:629-32.

50. Rofstad EK. Radiation response of the cells of a human malignant melanoma xenograft. Effect of hypoxic cell radiosensitizers. Radiat Res 1981;87:670-83.

51. Palcic B, Faddegon B, Skarsgard LD. The effect of misonidazole as a hypoxic radiosensitizer at low dose. Radiat Res 1984;100:340-7.

52. Wittenborn TR, Horsman MR. Targeting tumour hypoxia to improve outcome of stereotactic radiotherapy. Acta Oncol 2015;54:1385-92.

53. Nori D, Cain JM, Hilaris BS, Jones WB, Lewis JL Jr. Metronidazole as a radiosensitizer and high-dose radiation in advanced vulvovaginal malignancies, a pilot study. Gynecol Oncol 1983;16:117-28.

54. Schlaff CD, Krauze A, Belard A, O'Connell JJ, Camphausen KA Bringing the heavy: carbon ion therapy in the radiobiological and clinical context. Radiat Oncol 2014;9:88.

55. Schuler E, Trovati S, King G, et al. Experimental platform for ultra-high dose rate FLASH irradiation of small animals using a clinical linear accelerator. Int J Radiat Oncol Biol Phys 2016 Sep 20. [Epub]. http://dx.doi.org/10.1016/j.ijrobp.2016.09.018.

56. Favaudon V, Caplier L, Monceau V, et al. Ultrahigh dose- 
rate FLASH irradiation increases the differential response between normal and tumor tissue in mice. Sci Transl Med 2014;6:245ra93.

57. Palmer GM, Vishwanath K, Dewhirst MW. Application of optical imaging and spectroscopy to radiation biology. Radiat Res 2012;177:365-75.

58. Vishwanath K, Klein D, Chang K, Schroeder T, Dewhirst MW, Ramanujam N. Quantitative optical spectroscopy can identify long-term local tumor control in irradiated murine head and neck xenografts. J Biomed Opt 2009;14:054051.

59. Park J, Lee J, Kwag J, et al. quantum dots in an amphiphilic polyethyleneimine derivative platform for cellular labeling, targeting, gene delivery, and ratiometric oxygen sensing. ACS Nano 2015;9:6511-21.

60. Kersey FR, Zhang G, Palmer GM, Dewhirst MW, Fraser CL.
Stereocomplexed poly(lactic acid)-poly(ethylene glycol) nanoparticles with dual-emissive boron dyes for tumor accumulation. ACS Nano 2010;4:4989-96.

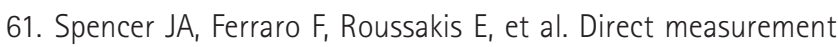
of local oxygen concentration in the bone marrow of live animals. Nature 2014;508:269-73.

62. Prasad P, Gordijo CR, Abbasi AZ, et al. Multifunctional albumin-MnO(2) nanoparticles modulate solid tumor microenvironment by attenuating hypoxia, acidosis, vascular endothelial growth factor and enhance radiation response. ACS Nano 2014;8:3202-12.

63. Song $X$, Feng L, Liang $C$, Yang $K_{1}$ Liu Z. Ultrasound triggered tumor oxygenation with oxygen-shuttle nanoperfluorocarbon to overcome hypoxia-associated resistance in cancer therapies. Nano Lett 2016;16:6145-53. 